\title{
Application of Zadoff-Chu Sequences in Throughput Balancing Control of Self-organizing Long Term Evolution Communication Network
}

\author{
Shengrong Lu, ${ }^{1}$ Yang-Han Lee, ${ }^{2 *}$ Yi-Lun Chen, ${ }^{2}$ Hsien-Wei Tseng, ${ }^{1 * *}$ \\ Chun-Chi Chen, ${ }^{3}$ Jhih-Hong Chen, ${ }^{4}$ Hen-Wai Tsao, ${ }^{4}$ and Jing-Shown $\mathrm{Wu}^{4}$ \\ ${ }^{1}$ College of Mathematics and Information Engineering, Longyan University, Fujian 364012, China \\ ${ }^{2}$ Department of Electrical and Computer Engineering, Tamkang University, New Taipei City 251301, Taiwan \\ ${ }^{3}$ School of Life Sciences, Longyan University, Longyan, Fujian 364012, China \\ ${ }^{4}$ Department of Electrical Engineering, National Taiwan University, Taipei City 10617, Taiwan
}

(Received October 21, 2020; accepted March 22, 2021)

Keywords: SON, Zadoff-Chu sequence, QPSK, 16 QAM, 64 QAM

In this paper, we consider the implementation of different Zadoff-Chu sequences to different modulation levels in communication systems with multiple modulation levels. The base station of the system can dynamically estimate the number of users in each modulation level by detecting the Zadoff-Chu sequences it receives. The base station then calculates the throughput of each modulation level and determines if any modulation level is saturated. If a modulation level is saturated, then the base station implements an additional mechanism on the users in the modulation level with the largest throughput. This mechanism makes users enter a waiting period before allowing further data transmissions so as to mitigate or reduce the base station load to accomplish the tasks and functions of the self-organizing network.

\section{Introduction}

In the 4G network, network operators apply a self-organizing network (SON) scheme to reduce the manual involvement and operational cost when there are several coexisting networks to reduce the complexity of the resulting overall network system. ${ }^{(1-7)}$

The SON is an important feature of new networks such as long term evolution (LTE) and IEEE 802.16. It supports the automatic configuration and optimization of cells, reduces the complexity and cost of manually setting the network parameters, and optimizes the network coverage, capacity, and mobile load balance. The SON also adjusts the parameters of the base station according to the interference level experienced by the base station to improve the network performance. For the SON to function properly, it requires information such as the number of users and their distributions in the cell; such information has a great impact on the allocation of resources in the base station. Therefore, how to dynamically detect the number of users associated with a base station has become an important issue.

\footnotetext{
*Corresponding author: e-mail: yhleepp@gmail.com

** Corresponding author: e-mail: hsienwei.tseng@gmail.com

https://doi.org/10.18494/SAM.2021.3177
} 
The coexistence of different networks increases the complexity of a network. In the era of the Internet of Things (IoT), wireless sensor networks are very important and can be applied to security monitoring, logistics tracking, home care, and other fields. A large number of sensors are deployed in the monitoring area for data collection, analysis, and real-time monitoring. Thus, the number of devices that the LTE SON can accommodate is very important. To realize some autonomous functions of wireless networks, reduce manual operations, and reduce operating costs in future networks, network operators have proposed the concept of SONs. A SON is an important function of LTE technology. This function supports the automatic configuration and optimization of new Fengcao cells and other important features, simplifying the process of manually configuring parameters in network construction and reducing the difficulty and cost of construction. This function is also used for the optimization of network coverage and capacity, mobile load balancing optimization, cell interference coordination, adaptive adjustment of parameters, and optimization of network performance.

In the LTE specifications, Zadoff-Chu sequences have been widely selected as the reference signal in many applications, such as the primary synchronization signal (PSS), the uplink reference signal, and the reference signal in the random access channel and also in the physical uplink control channel (PUCCH). ${ }^{(1-7)}$ In this paper, we consider a wireless communication system where the users in the base station are classified according to their modulation format. Three modulations are considered, namely, QPSK, 16 QAM, and 64 QAM. A different Zadoff-Chu sequence is implemented for each modulation format and the base station decodes the received Zadoff-Chu sequences that are transmitted from users in different modulation levels. From the decoded result, the base station can detect the number of users distributed in every modulation level and, consequently, it can determine the resulting throughput of users in each modulation level. Finally, the base station can detect whether it is saturated, i.e., having too many users in its coverage area. If the number of users in the base station is saturated, it will implement an additional mechanism for each user in the modulation level having the largest throughput to mitigate or reduce its load. In this way, the base station can accomplish the functions and application of the SON.

In this paper, we study the assignment of a different Zadoff-Chu sequence to every modulation level in communication systems with multiple modulation levels, which is a technical design for wireless sensor networks, making the results applicable to sensing systems. The organization of this paper is as follows. In Sect. 2, Zadoff-Chu sequences and their characteristics are introduced, the autocorrelation of a Zadoff-Chu sequence is calculated, and the cross-correlation of two Zadoff-Chu sequences is simulated and discussed. In Sect. 3, the system architecture is introduced, the assignment of a Zadoff-Chu sequence to each user is implemented, the system throughput is then calculated, and a scheme is designed to mitigate or reduce the system throughput when the system is overloaded. A conclusion is given in Sect. 4.

\section{Zadoff-Chu Sequences}

A Zadoff-Chu sequence is defined as ${ }^{(8,9)}$ 


$$
u_{r}(N)=\left\{\begin{array}{l}
e^{-j \frac{r \pi n^{2}}{N_{z c}}}, n=0,1, \ldots,\left(N_{z c}-1\right), \quad \text { when } N_{z c} \text { is even } \\
e^{-j \frac{r \pi n(n+1)}{N_{z c}}}, n=0,1, \ldots,\left(N_{z c}-1\right), \text { when } N_{z c} \text { is odd }
\end{array}\right.
$$

where $N_{z c}$ is the Zadoff-Chu sequence length and $r$ is the sequence root.

A Zadoff-Chu sequence has the following important properties: (1) the sequence length $N_{z c}$ of any Zadoff-Chu sequence and the amplitude of the sequence are definite and deterministic values, (2) if $N_{z c}$ is a prime number, then the period of the sequence is an integer $N_{z c}$, and $u_{r}\left(n+N_{z c}\right)=u_{r}(n)$, (3) for a fixed number $r$ relatively prime to $N_{z c}$, the Zadoff-Chu sequence has an ideal periodic autocorrelation property, i.e., the periodic autocorrelation is zero for all nonzero time shifts, ${ }^{(10)}$ (4) if the sequence length $N_{z c}$ is a prime number, then there are $\left(N_{z c}-1\right)$ different sequences with a periodic cross-correlation value of $1 / \sqrt{N_{z c}}$ between any two sequences regardless of their time shift, ${ }^{(10)}$ and (5) the discrete Fourier transform of a Zadoff-Chu sequence is another Zadoff-Chu sequence. ${ }^{(11-15)}$

The autocorrelation and cross-correlation functions of the Zadoff-Chu sequence are defined as $x_{0}$ and $x_{j}$, respectively, as follows: ${ }^{(10)}$

$$
\begin{aligned}
& x_{0}=\sum_{n=0}^{N_{z c}-1} u_{r_{1}}(n) u_{r_{2}}^{*}(n), \\
& x_{j}=\sum_{n=0}^{N_{z c}-j-1} u_{r_{1}}(n) u_{r_{2}}^{*}(n+j)+\sum_{n=N_{z c}-j}^{N_{z c}-1} u_{r_{1}}(n) u_{r_{2}}^{*}\left(n+j-N_{z c}\right), j=1,2, \ldots,\left(N_{z c}-1\right),
\end{aligned}
$$

where $j$ is the shift index of the Zadoff-Chu sequence.

When $r_{1}=r_{2}$, the above function becomes the autocorrelation function of the Zadoff-Chu sequence; when $r_{1} \neq r_{2}$, it becomes the cross-correlation function of the Zadoff-Chu sequences.

The peak value of the autocorrelation function $x_{0}$ of a Zadoff-Chu sequence with length $N_{z c}$ as defined above is $N_{z c}$; in other words, the peak value $x_{0}$ of Zadoff-Chu sequences of different lengths will be different. For this reason, to properly handle Zadoff-Chu sequences of different lengths, it is necessary to normalize the autocorrelation function of Zadoff-Chu sequences of different lengths to have the same normalized value and also to make the cross-correlation function of the Zadoff-Chu sequence satisfy property (4) above. Consequently, the above equations of the autocorrelation function and cross-correlation function are modified to the following.

$$
\begin{aligned}
& x_{0}=\frac{1}{N_{z c}} \sum_{n=0}^{N_{z c}-1} u_{r_{1}}(n) u_{r_{2}}^{*}(n) \\
& x_{j}=\frac{1}{N_{z c}}\left[\sum_{n=0}^{N_{z c}-j-1} u_{r_{1}}(n) u_{r_{2}}^{*}(n+j)+\sum_{n=N_{z c}-j}^{N_{z c}-1} u_{r_{1}}(n) u_{r_{2}}^{*}\left(n+j-N_{Z C}\right)\right], j=1,2, \ldots,\left(N_{z c}-1\right)
\end{aligned}
$$


When the root $r$ and the sequence length $N_{z c}$ of the Zadoff-Chu sequence are $r=2$ and $N_{z c}=55$, i.e., relatively prime, its autocorrelation function has the values shown in Fig. 1. On the other hand, when $r=11$ and $N_{z c}=55$, i.e., not relatively prime, the autocorrelation function has the values shown in Fig. 2.

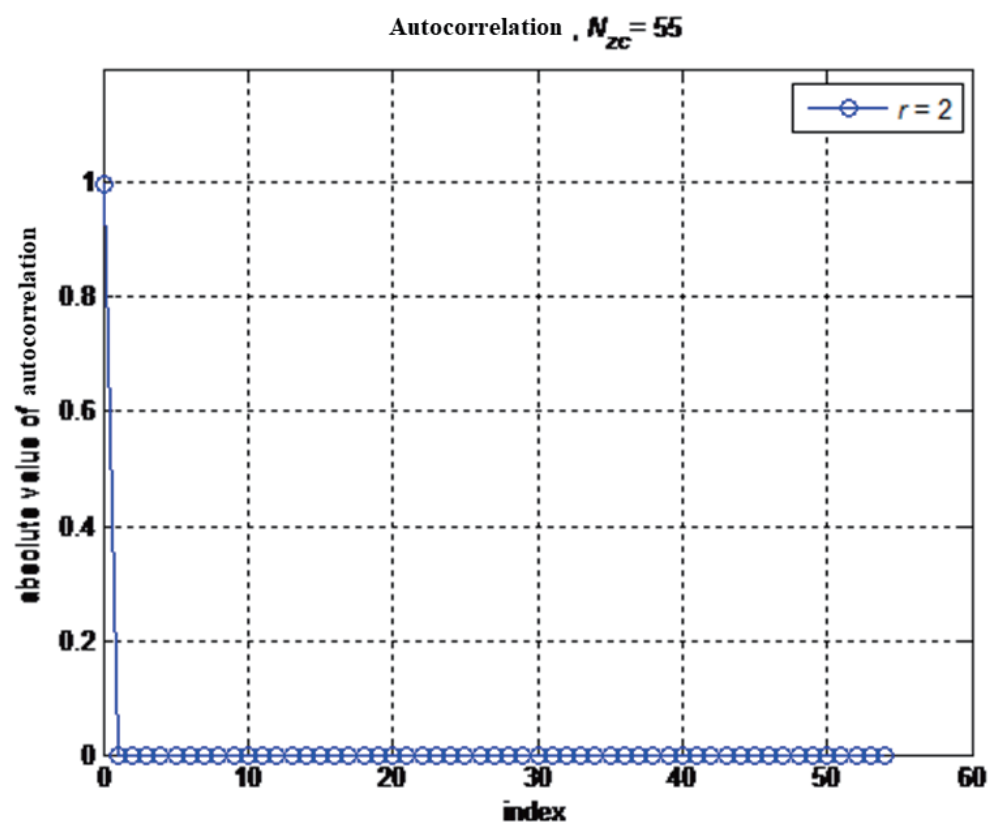

Fig. 1. (Color online) Autocorrelation function with $r=2, N_{z c}=55$, i.e., the root of the Zadoff-Chu sequence and its sequence length are relatively prime.

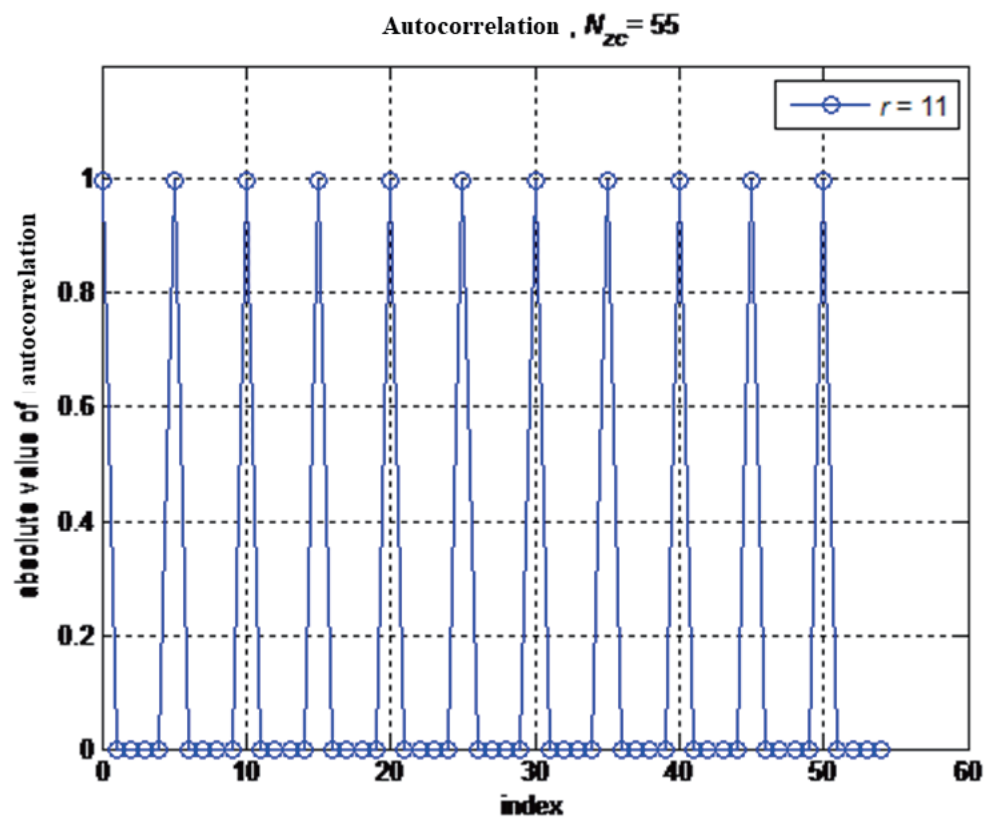

Fig. 2. (Color online) Autocorrelation function with $r=11, N_{z c}=55$, i.e., the root of the Zadoff-Chu sequence and its sequence length are not relatively prime. 
An example of the cross-correlation function of two Zadoff-Chu sequences with the same length $N_{z c}$ but with different roots $r_{1}, r_{2}\left(r_{1} \neq r_{2}\right)$ and $\left|r_{1}-r_{2}\right|$ and $N_{z c}$ relatively prime is shown in Fig. 3, where $r_{1}=2, r_{2}=4$, and $N_{z c}=55$. Figure 4 shows an example of the cross-correlation function of two Zadoff-Chu sequences when $\left|r_{1}-r_{2}\right|$ and $N_{z c}$ are not relatively prime, i.e., $r_{1}=2$, $r_{2}=7$, and $N_{z c}=55$.

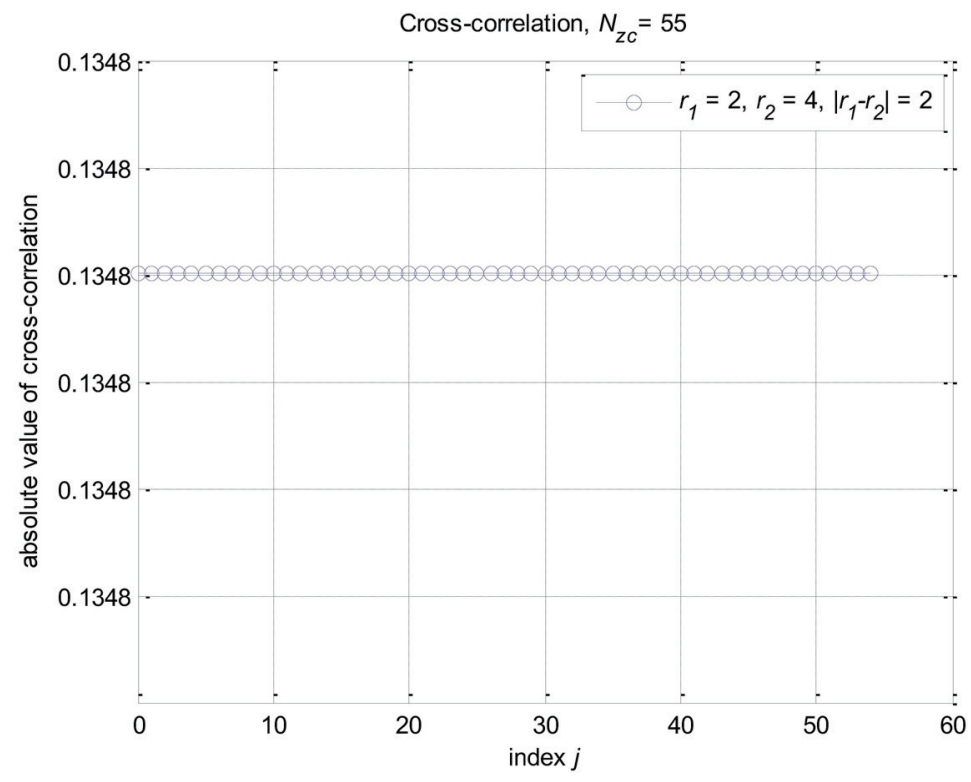

Fig. 3. (Color online) Cross-correlation function with $r_{1}=2, r_{2}=4$, and $N_{z c}=55$ for two Zadoff-Chu sequences with $N_{z c}$ and $\left|r_{1}-r_{2}\right|$ relatively prime.

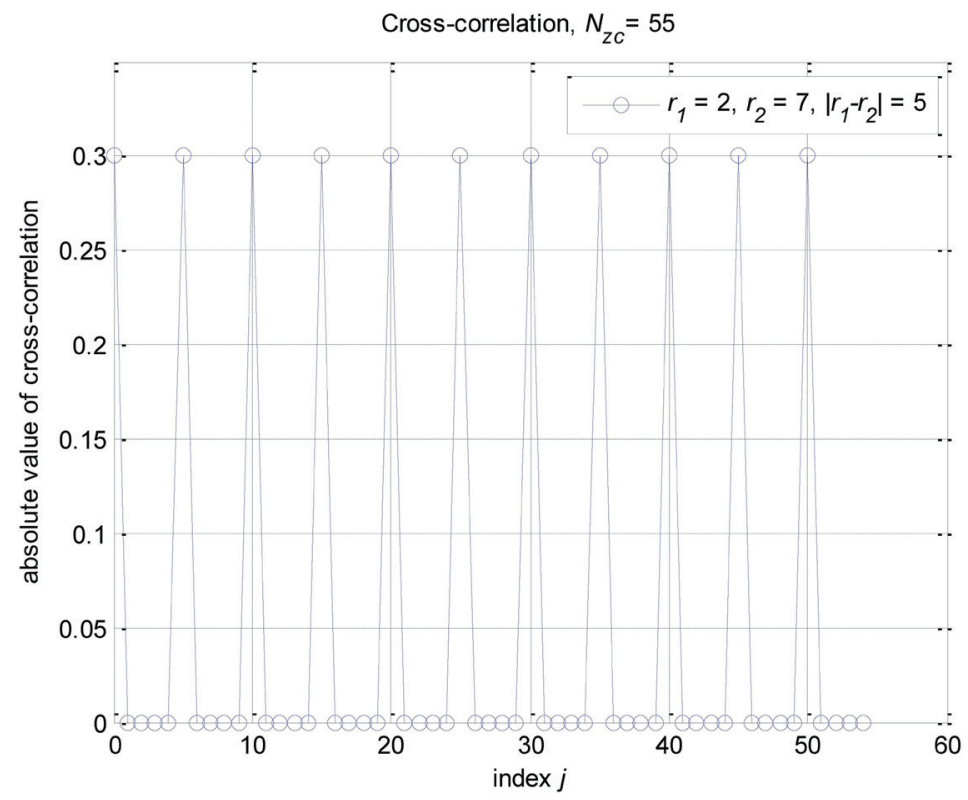

Fig. 4. (Color online) Cross-correlation function with $r_{1}=2, r_{2}=7$, and $N_{z c}=55$ for two Zadoff-Chu sequences with $N_{z c}$ and $\left|r_{1}-r_{2}\right|$ not relatively prime. 
Figure 3 verifies property (4) as above, that is, when two Zadoff-Chu sequences have the same length but different root values and the difference between their roots and the sequence length are relatively prime, then their cross-correlation value is a constant $1 / \sqrt{N_{z c}}$. Moreover, for two Zadoff-Chu sequences of the same length and different root values, if the difference between their two roots is relatively prime to the sequence length, then the longer the sequence length, the smaller the cross-correlation constant value. This suggests that for two Zadoff-Chu sequences, if we select suitable roots and sequence lengths, then we can achieve a smaller crosscorrelation value.

\section{System Structure and Performance}

Suppose the number of users in a base station is $x$ and we classify the users into three levels according to their modulation formats: Levels A, B, and C for QPSK, 16 QAM, and 64 QAM modulations, respectively. Suppose Levels $\mathrm{A}, \mathrm{B}$, and $\mathrm{C}$ have $x_{A}, x_{B}$, and $x_{C}$ users, respectively, with $x_{A}+x_{B}+x_{C}=x$. Assuming $x \bmod 3=0$, where mod denotes the remainder of the modulus operation, then there are $x / 3$ users in each modulation level. If $x \bmod 3=1$, then the number of users in Level A will be $x_{A}=$ floor $(x / 3)+1$, where floor $(x)$ is the largest integer less than $x$, and the numbers of users in Levels $\mathrm{B}$ and $\mathrm{C}$ will be $x_{B}=x_{C}=$ floor $(x / 3)$. If $x \bmod 3=2$, then the numbers of users in Levels $\mathrm{A}$ and $\mathrm{B}$ will be $x_{A}=$ floor $(x / 3)+1$ and $x_{B}=$ floor $(x / 3)+1$, respectively, and the number of users in Level C will be $x_{C}=$ floor $(x / 3)$.

When the number of users in the base station changes from $x$ to $y$, then the number of users in each modulation level will be changed accordingly; consequently, the base station needs some measures to calculate the number of users in each modulation level. Similarly, let the Zadoff-Chu sequences implemented in Levels $\mathrm{A}, \mathrm{B}$, and $\mathrm{C}$ be $u r_{A}, u r_{B}$, and $u r_{C}$, respectively. The base station receives a signal $C_{r}$ and performs the decoding process to calculate the number of users in each modulation level. As shown in Fig. 5, the base station receives a signal $C_{r}=x_{A}{ }^{*} P_{A}{ }^{*} u r_{A}+$ $x_{B}{ }^{*} P_{B}{ }^{*} u_{r B}+x_{C}{ }^{*} P_{C}{ }^{*} u_{r C}$ ( ${ }^{*}$ represents the convolution function), performs the cross-correlations of $C_{r}$ with respect to $\left(\gamma_{A}{ }^{*} u_{r A}\right),\left(\gamma_{B}{ }^{*} u_{r B}\right)$, and $\left(\gamma_{C}{ }^{*} u_{r C}\right)$, generates the cross-correlation values $x_{0}$, and takes their integer values to estimate the number of users in each level $x_{K}$. By defining $K=\mathrm{A}, \mathrm{B}$, or C, $u_{r K}$ is the Zadoff-Chu sequence in the $K$ th level with root $r_{K} ; P_{K}$ is the average signal power received by the base station from users in level $K$; and $\gamma_{A}, \gamma_{B}$, and $\gamma_{C}$ are the scaling factors for the $C_{r}$ cross-correlation values, which are the reciprocal values of the average received signal powers $P_{K}$.

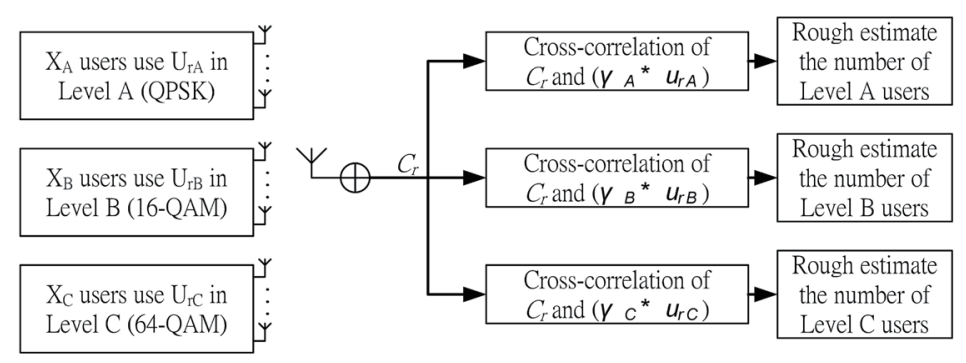

Fig. 5. System flow chart: three different Zadoff-Chu sequences with three power levels. 
The system parameters used in our system simulation are as follows:

- Base station power: $30 \mathrm{dBm}(1 \mathrm{~W})$.

- Base station radius: $1050 \mathrm{~m}$. This radius is calculated assuming the bit error rate (BER) set at $10^{-3}$ and a signal-to-noise ratio of $-0.46 \mathrm{~dB}$.

- Channel model: COST-231 Hata.

The modified Hata path loss model has the form

$$
P L_{\text {urban_Macro }}[d B]=32.29+38.35 \log _{10}(d)+26 \log _{10}(f / 2) \text {, }
$$

where $d$ is the transmission distance between the transmitter and receiver in meters and $f$ is the carrier frequency in MHz. The antenna heights for the base station and MS are 10 and $1.5 \mathrm{~m}$, respectively, and the carrier frequency is $2 \mathrm{GHz}$.

The following LTE specifications are used:

(a) Bandwidth: $10 \mathrm{MHz}$.

(b) Peak data rates: QPSK (14.4 Mbps), 16 QAM (28.8 Mbps), and 64 QAM (43.2 Mbps).

(c) Data rates considered in the simulation: QPSK (256 kbps), 16 QAM (512 kbps), and 64 QAM

(768 kbps).

(d) Thirty users are uniformly distributed in the coverage range of a $1 \mathrm{~W}$ base station or

(d') Eighty users are uniformly distributed in the coverage range of a $1 \mathrm{~W}$ base station.

In (d), the 30 users are uniformly distributed in the coverage area of the $1 \mathrm{~W}$ base station, and the simulated information between the base station and these 30 users is tabulated in Table 1. In Table 1, the entries in the Range column are the distances between the base station and the users; the entries in the Power column are the powers of the signals received by the base station; and the entries in the SNR column are the SNR power ratios of the signals received by the base station. After averaging all the received signal powers in the same modulation level, the resulting

Table 1

Simulated information between the base station and 30 users.

\begin{tabular}{|c|c|c|c|c|c|c|c|c|c|}
\hline $\begin{array}{l}\text { MS } \\
\text { number }\end{array}$ & Range (m) & $\begin{array}{l}\text { Power } \\
(\mathrm{dBm})\end{array}$ & SNR & Level & $\begin{array}{l}\text { MS } \\
\text { number }\end{array}$ & Range (m) & $\begin{array}{l}\text { Power } \\
(\mathrm{dBm})\end{array}$ & SNR & Level \\
\hline 1 & 264 & -81 & 23 & $\mathrm{C}$ & 16 & 226 & -79 & 25 & C \\
\hline 2 & 496 & -92 & 12 & B & 17 & 954 & -103 & 1 & A \\
\hline 3 & 917 & -102 & 2 & A & 18 & 720 & -98 & 6 & A \\
\hline 4 & 965 & -103 & 1 & A & 19 & 906 & -102 & 2 & A \\
\hline 5 & 528 & -93 & 11 & B & 20 & 823 & -100 & 4 & A \\
\hline 6 & 602 & -95 & 9 & B & 21 & 742 & -98 & 6 & A \\
\hline 7 & 808 & -100 & 4 & A & 22 & 570 & -94 & 10 & B \\
\hline 8 & 864 & -101 & 3 & A & 23 & 278 & -82 & 22 & $\mathrm{C}$ \\
\hline 9 & 776 & -99 & 5 & A & 24 & 465 & -91 & 13 & B \\
\hline 10 & 446 & -90 & 14 & B & 25 & 549 & -93 & 11 & B \\
\hline 11 & 401 & -88 & 16 & $\mathrm{C}$ & 26 & 573 & -94 & 10 & B \\
\hline 12 & 751 & -99 & 5 & A & 27 & 603 & -95 & 9 & B \\
\hline 13 & 459 & -90 & 14 & B & 28 & 792 & -99 & 5 & A \\
\hline 14 & 757 & -99 & 5 & A & 29 & 915 & -102 & 2 & A \\
\hline 15 & 755 & -99 & 5 & A & 30 & 974 & -103 & 1 & A \\
\hline
\end{tabular}


average powers in the modulation levels are $P_{A}(-100.3 \mathrm{dBm}), P_{B}(-92.7 \mathrm{dBm})$, and $P_{C}(-82.5 \mathrm{dBm})$ for Levels $\mathrm{A}, \mathrm{B}$, and $\mathrm{C}$, respectively.

The Zadoff-Chu sequence selected in the simulation has length 61 with root values 25,29 , and 32; the scaling factors $\gamma_{A}, \gamma_{B}$, and $\gamma_{C}$ are selected as the inverses of the average received powers in each level, i.e., they are $1 / 100.3,1 / 92.7$, and $1 / 82.5$, respectively, from the above calculated received signal power at the base station. The calculated number of users in each level is listed in Table 2.

After the number of users is determined in each level, we can calculate the throughput of each level. The throughput in Level A is calculated as $(16 \times 256)=4.096 \mathrm{Mbps}$, with similar throughputs in Levels B and C of 5.632 and $2.304 \mathrm{Mbps}$, respectively. The remaining capacity in the base station is calculated from the peak data rate in each level, and the remaining capacity in this base station is calculated in the following way:

(1) $\mathrm{MC}=$ Remaining capacity after satisfying data traffic of Level C:

Peak data rate (64 QAM) - throughput (64 QAM)

(2) $\mathrm{MB}=$ Remaining capacity after satisfying data traffic of Level B:

MC*4/6 - throughput (16 QAM)

(3) $\mathrm{MA}=$ Final remaining capacity in base station:

MB*2/4 - throughput (QPSK)

From the above simulation result with a transmitting power of $1 \mathrm{~W}$ and 30 users, we calculate the remaining capacity in the base station as $6.72 \mathrm{Mbps}$. When the remaining capacity in the base station is larger than zero, the base station can serve more users after satisfying the traffic requirements of the 30 users distributed in Levels $\mathrm{A}, \mathrm{B}$, and $\mathrm{C}$.

Next, we simulate the situation with 80 users uniformly distributed in the base station with a transmitting power of $1 \mathrm{~W}$ to find the traffic capacity in each level. The sequence length of the Zadoff-Chu sequence selected is 61 and the root values selected in Levels A, B, and C are 25, 29, and 32, respectively. Following a similar process to that for the case of 30 users, we obtain the average received signal powers of $P_{A}(-101 \mathrm{dBm}), P_{B}(-94.6 \mathrm{dBm})$, and $P_{C}(-78 \mathrm{dBm})$ with scaling factors of $\gamma_{A}(1 / 101), \gamma_{B}(1 / 94.6)$, and $\gamma_{C}(1 / 78)$ for the three levels. The number of users in each modulation level obtained by simulation is listed in Table 3.

Table 2

Calculated numbers of users in each level when 30 users are uniformly distributed in a cell of $1050 \mathrm{~m}$ radius with $1 \mathrm{~W}$ transmitting power.

\begin{tabular}{|c|c|c|c|c|c|c|c|c|}
\hline \multicolumn{3}{|c|}{ Numbers of users } & \multicolumn{3}{|c|}{$\begin{array}{c}\text { BS: Cross-correlation of } C_{r} \text { and } \\
\left(\gamma_{K}^{*} u_{K}\right) \text { for } K=\mathrm{A}, \mathrm{B}, \mathrm{C}\end{array}$} & \multicolumn{3}{|c|}{$\begin{array}{c}\text { BS: Rough estimate of number of leve } \\
K \text { users for } K=\mathrm{A}, \mathrm{B}, \mathrm{C}\end{array}$} \\
\hline Level A & Level B & Level C & Level A & Level B & Level C & Level A & Level B & Level C \\
\hline 16 & 10 & 4 & 16.818 & 11.813 & 3.315 & 16 & 11 & 3 \\
\hline
\end{tabular}

Table 3

Calculated numbers of users in each level when 80 users are uniformly distributed in a cell of $1050 \mathrm{~m}$ radius with $1 \mathrm{~W}$ transmitting power.

\begin{tabular}{|c|c|c|c|c|c|c|c|c|}
\hline \multicolumn{3}{|c|}{ Numbers of users } & \multicolumn{3}{|c|}{$\begin{array}{c}\text { BS: Cross-correlation of } \mathrm{C}_{\mathrm{r}} \text { and } \\
\left(\gamma_{K}^{*} u_{K}\right) \text { for } K=\mathrm{A}, \mathrm{B}, \mathrm{C}\end{array}$} & \multicolumn{3}{|c|}{$\begin{array}{c}\text { BS: Rough estimate of number of level } \\
\qquad K \text { users for } K=\mathrm{A}, \mathrm{B}, \mathrm{C}\end{array}$} \\
\hline Level A & Level B & Level C & Level A & Level B & Level C & Level A & Level B & Level C \\
\hline 48 & 16 & 16 & 48.943 & 21.712 & 11.575 & 48 & 21 & 11 \\
\hline
\end{tabular}


Following the same calculation process as above, after determining the number of users in each modulation, we determine the throughputs to be 12.288, 10.752, and 8.448 Mbps in Levels $\mathrm{A}, \mathrm{B}$, and $\mathrm{C}$, respectively. The remaining capacity of this base station is then $-6.08 \mathrm{Mbps}$, meaning that the base station cannot provide sufficient capacity to meet the traffic requirements of these 80 users.

The base station implements an extra mechanism of user load mitigation or reduction in one of the modulation levels when it cannot provide services to meet the users' requirements in the coverage range. The base station sends an extra Zadoff-Chu sequence, the Zadoff-Chu waiting sequence $u_{r S}$, to the modulation level that generates the largest throughput. When each user in the modulation level with the largest throughput receives this waiting sequence $u_{r S}$ in its uplink, it will send not only its regular Zadoff-Chu sequence but also this waiting Zadoff-Chu sequence to the base station. The users in this modulation level then enter a random waiting mode, with their control channel still linked to the base station. After waiting for a random time interval, users in this modulation level will again be able to transmit their data to the base station. The system equipped with this load mitigation/reduction mechanism has the functional block diagram shown in Fig. 6.

As shown in Fig. 6, each user in Level A transmits not only the Zadoff-Chu sequence that will be used by the base station for its cross-correlation calculation but also the waiting sequence $u_{r S}$ to the base station. The sequence length of the Zadoff-Chu sequence considered is 61 and the root values in Levels A, B, and C are 25, 29, and 32, respectively, whereas the root value of the Zadoff-Chu waiting sequence is 22 . The simulated number of users in each modulation level for this case is listed in Table 4.

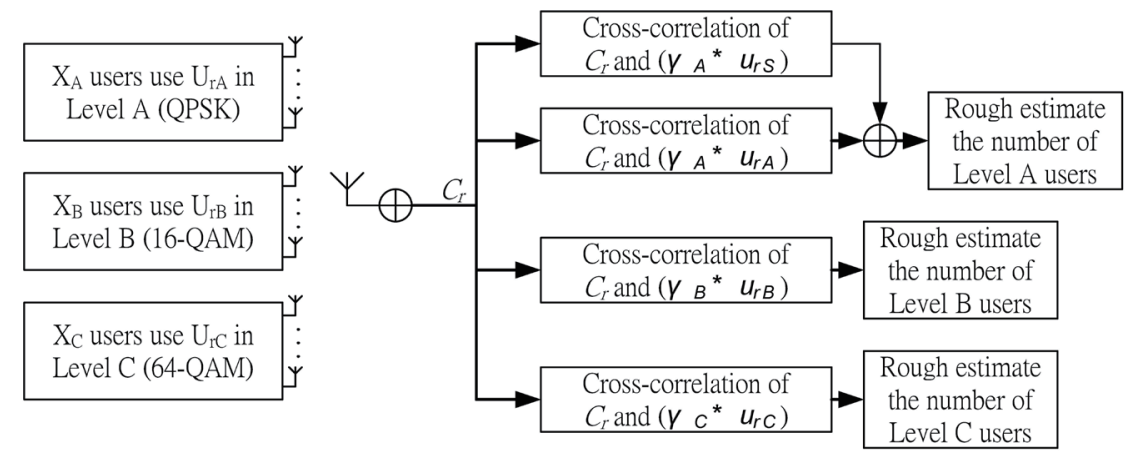

Fig. 6. Functional block diagram of system with load mitigation/reduction mechanism implemented in Level A.

Table 4

Calculated number of users in each level after load mitigation/reduction process when 80 users are uniformly distributed in a cell of $1050 \mathrm{~m}$ radius with $1 \mathrm{~W}$ transmitting power.

\begin{tabular}{|c|c|c|c|c|c|c|c|c|c|c|}
\hline \multicolumn{3}{|c|}{ Numbers of users } & \multicolumn{4}{|c|}{$\begin{array}{l}\text { BS: Cross-correlation of } C_{r} \text { and }\left(\gamma_{K}{ }^{*} u_{K}\right) \\
\text { for } K=\mathrm{A}, \mathrm{B}, \mathrm{C}\end{array}$} & \multicolumn{4}{|c|}{$\begin{array}{c}\text { BS: Rough estimate of number of level } K \\
\text { users for } K=\mathrm{A}, \mathrm{B}, \mathrm{C}\end{array}$} \\
\hline Level A & Level B & Level C & $\begin{array}{l}\text { Level A } \\
(r=25)\end{array}$ & $\begin{array}{l}\text { Level A } \\
(r=22)\end{array}$ & Level B & Level C & $\begin{array}{l}\text { Level A } \\
(r=25)\end{array}$ & $\begin{array}{l}\text { Level A } \\
(r=22)\end{array}$ & Level B & Level C \\
\hline 48 & 16 & 16 & 44.882 & 43.488 & 17.748 & 17.940 & 44 & 43 & 17 & 17 \\
\hline
\end{tabular}


After the base station determines the number of users in each modulation level, it calculates the current throughput in each modulation level. In this case, Level A has one user (|Level A $(r=25)$-Level A $(r=22) \mid)$, and Levels B and $\mathrm{C}$ each have 17 users. The throughputs in Levels A, B, and C are $0.256,8.704$, and 13.059 Mbps, respectively. In this environment of 80 users uniformly distributed in a base station with $1 \mathrm{~W}$ transmitting power, after the users in Level A enter the waiting mode, the remaining capacity in the base station is $5.439 \mathrm{Mbps}$, meaning that the base station has extra capacity to provide a service to more users.

\section{Conclusion}

In the LTE specifications, many system designs in the control channel and traffic channel are based on the applications of the characteristics of Zadoff-Chu sequences. In this paper, we discussed in detail the characteristics of Zadoff-Chu sequences, then we selected and implemented three suitable Zadoff-Chu sequences for users in three different modulation levels. In this design, the users are divided into three modulation levels depending on their modulation format, then the base station acquires a rough estimate of the number of users in each modulation level from its received and demodulated user signals. An advantage of our implementation using different Zadoff-Chu sequences for different modulation levels is that the base station can detect when its capacity becomes saturated. This triggers a mechanism to notify the users in the modulation level with the largest capacity to enter a waiting mode for their data transmissions so that the base station can increase its capacity and avoid saturation.

\section{Acknowledgments}

This work was supported by Longyan University's Qi Mai Science and Technology Innovation Fund Project of Longyan City (2017QM0201, 2018LYQM0202); Longyan University's Qi Mai Science and Technology Innovation Fund Project of Liancheng ([2018] No.132) and Shanghang (2019SHQM05) Counties; Longyan and Longyan University's Research and Development Team Fund ((2018)8); Great Project of Production, Teaching, and Research of Fujian Provincial Science and Technology Department (2019H6023); and the Ministry of Science and Technology, Taiwan, project of MOST 109-2321-B-001-013 and the Ministry of Education, Taiwan, project of "Agriculture Artificial Intelligence Internet of Things (AgAIoT)".

\section{References}

1 Y. Wen, W. Huang, and Z. P. Zhang: Proc. 2006 IEEE Information Theory Workshop (IEEE, Chengdu, 2006) 544-547.

2 M. M. Mansour: Proc. 2009 IEEE Global Telecommunications Conf. (IEEE, 2009) 1-6.

3 Y. B. Tang and W. C. Ge: Proc. 2009 Asia-Pacific Conf. Information Processing (IEEE, 2009) 140-143.

4 Y. Yang, W. Che, N. Yan, X. Tan, and H. Min: Electron. Lett. 46 (2010) 376.

5 S. W. Kim and K. Y. Kim: Proc. 2009 11th Int. Conf. Advanced Communication Technology (IEEE, 2009) 1095-1100.

6 M. Lu, B. Chen, X. L. Hou, X. Zhang, and D. M. Luo: Proc. 2008 IEEE 68th Vehicular Technology Conf. (IEEE, 2008) 1-5.

7 T. H. Pham, Y. C. Liang, A. Nallanathan, and G. H. Krishn: IEEE Signal Process. Lett. 16 (2009) 200. 
8 D. Chu: IEEE Trans. Inf. Theory 18 (1972) 531

9 R. C. Heimille: IRE Trans. Inf. Theory 7 (1961) 254.

10 F. Khan: LTE for 4G Mobile Broadband: Air Interface Technologies and Performancem, 1st ed. (Cambridge University Press, 2009).

11 S. Beyme and C. Leung: Electron. Lett. 45 (2009) 46.

12 C. P. Li and W. C. Huang: IEEE Trans. Inf. Theory 53 (2007) 4221.

13 B. M. Popovic: Electron. Lett. 46 (2010) 502.

14 J. Blumenstein and M. Bobula: IEEE Commun. Lett. 22 (2018) 1006.

15 K. Tomimoto and R. Yamaguch: Proc. 2018 International Symposium on Antennas and Propagation (IEEE, 2018) $1-2$. 